\title{
The Analysis of the Location Algorithm for Mobile Targets Based on Mono-Station Passive Infrared Method
}

\author{
Shi-quan Zhang*, Jun Zeng \\ School of Science \\ Engineering University of CAPF \\ Xi'an 710086, China \\ * e-mail:zsquany@163.com
}

\author{
Shi-min Yin \\ College of Life and Environmental Science \\ Guilin University of Electronic Technology \\ Guilin 541004, China
}

\begin{abstract}
The passive infrared mono-station location algorithm for mobile targets due to stationary platform are investigated. The passive location physical model and related algorithm of mobile target are established. The algorithm in this paper can be effectively applied to the location for both uniformly accelerated mobile targets and those with varying acceleration. Numerical simulations verify the correctness and the feasibility of the algorithm and indicate that the relative ranging error is less than $5 \%$ of the slope distance from target to platform which completely satisfies the specification requirement of mono-station infrared passive location.
\end{abstract}

Keywords-mobile targets; infrared; mono-station passive location

\section{INTRODUCTION}

In modern warfare the aerial targets have significantly challenged the survival of weapons and military apparatus in the land. Once the signals radiating from the ground weapons and military apparatus are received by the enemy the ground target might be quickly attacked by anti-radiating missiles. If the ground systems are equipped with passive detecting and locating device under the prerequisite of efficient concealment, powerful countermeasure capability to the attacking targets can be achieved. Some location algorithms for aerial mobile targets on the basis of monostation passive infrared method have been reported[1-3]. New schemes of passive location were put forward in references [4-7] based on the principles of photoelectric imaging and image array measurement and passive location problem of mobile targets by non-mobile platform was explored. However, these schemes can not be applied to situations in which the target plane is not parallel to camera plane that the motion state and posture change. In this paper some innovations are made to the present scheme, appropriate model is established, a novel algorithm is proposed by real time renewing to the velocity and acceleration of the target to adapt to the variation of moving state of the target and some optimizations have been made to the algorithm.

\section{PHYSICAL MODELING AND ALGORITHM ANALYSIS}

The physical model for mono-station passive infrared location technique by stationary platform is shown in Fig.1. mono-station passive infrared location system is placed in a measurement station $C$ which is fixed in position on the ground or in slow motion The system can track the aerial threatened targets in a real time way. The mobile target is situated in point $A$ at the initial time, which moves at a speed of $v_{0}$ and is $R_{0}$ meters away from the measurement station. After a time interval $T$, the mobile target is situated at point $B$ and moves at a speed of $v_{l}$ and is $R_{l}$ meters away from the measurement station. The spanning angle of the target subtended to the station during this time interval is $\theta_{0}$. The passive infrared system which is composed of infrared focal plane array detectors in the measurement station make tracking and measurement to the target in a real-time way to obtain the information of azimuth and high-low angle of the target as well as the imaging information. Our purpose of study is to acquire the real-time measurement information at every sampling time such as information of azimuth and high-low angle, distance between target and measuring station as well as imaging information of the target by using the passive infrared system in the measuring station.

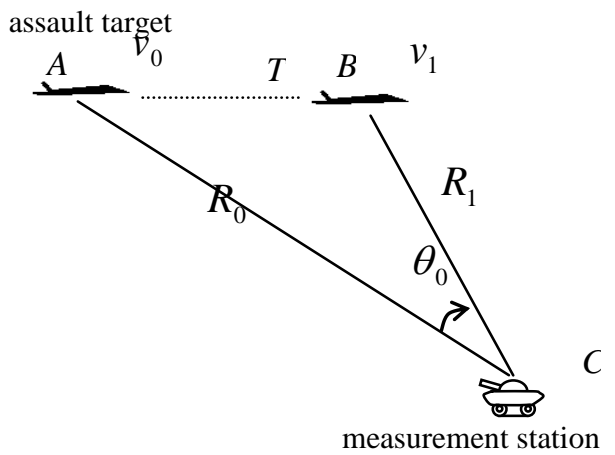

Fig. 1 physical modeling of mono-station of infrared passive localization

In terms of imaging theorem, we have the relations at time $B_{i}$ and $B_{i+1}$

$$
\frac{R_{i}}{f_{i}}=\frac{L \sin \varphi_{i}}{L_{i}}
$$




$$
\frac{R_{i+1}}{f_{i+1}}=\frac{L \sin \varphi_{i+1}}{L_{i+1}}
$$

From Equ. (1) and Equ. (2), we can obtain

$$
R_{i+1}=\frac{f_{i+1} \sin \varphi_{i+1} L_{i}}{f_{i} \sin \varphi_{i} L_{i+1}} R_{i}
$$

Ignoring the effect of the variation of the angle of course of the target, that is, setting $\sin \varphi_{i+1} / \sin \varphi_{i}=1$, we further have

$$
R_{i+1}=\frac{f_{i+1}}{f_{i}} \frac{L_{i}}{L_{i+1}} R_{i}
$$

Equ. (4) is a rudimentary location formula which is the same as reference [1] and [2]. This formula is unable to satisfy the precision requirement for locating mobile target due to the ignorance of the effect of the variation of the angle of course of the target.

We can also have the following relation from the model as shown in Fig.2

$$
\frac{\sin \varphi_{i+1}}{\sin \varphi_{i}}=\frac{R_{i}}{R_{i+1}}
$$

Combining Equ. (3) and Equ. (5), we have

$$
R_{i+1}=\sqrt{\frac{f_{i+1} L_{i}}{f_{i} L_{i+1}}} R_{i}
$$

Equ. (6) is the location formula we want.

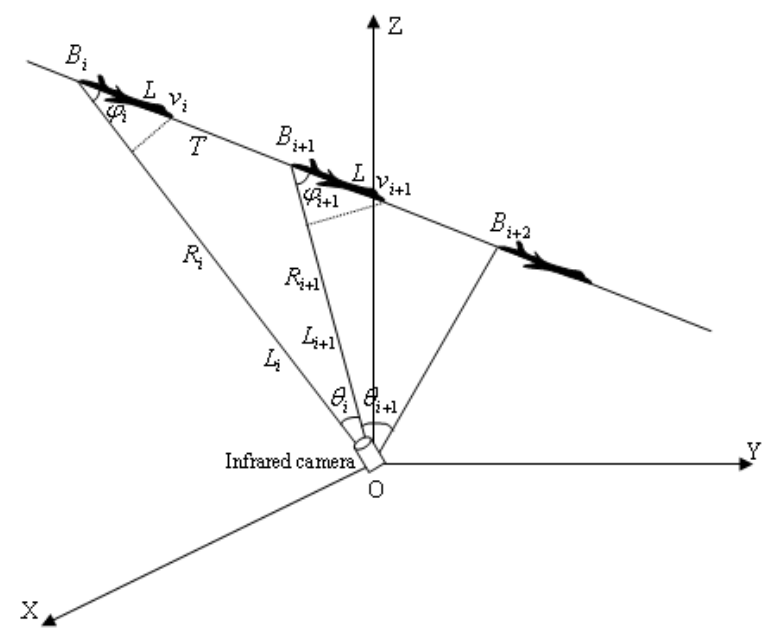

Fig. 2 The mathematical model of infrared mono-station passive localization

Based on the derivation process to the above formula, we can see clearly that our algorithm can overcome the passive effects of the pseudo-gesture variation of the mobile target on location precision since we take account of the information of the variation of the angle of course of the target. In addition, the variation of the moving state is included in the variation of the imaging size. Therefore, the above location formula can efficiently adapt to the situation of the variation of the moving state of mobile target. In other words, the location algorithm can be applied to the case in which the mobile target travels with constant acceleration and with changing acceleration.

As to radial motion target, i.e., the moving direction of mobile target is the same as that of the line of sight of infrared camera, the angle of course of the target at the adjacent moment are approximately equal to zero. Taking limit to Equ. (3), we can obtain the location formula for radial motion target as follows

$$
R_{i+1}=\lim _{\substack{\varphi_{i+1} \rightarrow 0 \\ \varphi_{i} \rightarrow 0}}\left(\frac{\sin \varphi_{i+1}}{\sin \varphi_{i}}\right) \frac{f_{i+1}}{f_{i}} \frac{L_{i}}{L_{i+1}} R_{i}=\frac{f_{i+1}}{f_{i}} \frac{L_{i}}{L_{i+1}} R_{i}
$$

where $L_{i}, L_{i+1}$ can be viewed as the maximum size of the image of the target taken on the infrared focal plane.

Equ. (6) can be used for locating the oblique target and Equ. (7) can be used for locating the radial target.

\section{THE ANALYSIS OF RANGING ERROR}

In accordance with Equ. (6), the error of radial distance $R_{i+1}$ is composed of that of the terms $f_{i}, f_{i+1}, L_{i} 、 L_{i+1}, R_{i}$, in which the error caused by the variation of real-time focal length, $f_{i}$ and $f_{i+1}$, at every time belongs to the scope of systematic error and we do not want to discuss. The error or $R_{i+1}$ is composed of the measurement error term of image parameter, $L_{i}$ and $L_{i+1}$, and the error term of the initial distance $R_{i}$ of the target.

The combined error of $R_{i+1}$ at present time is

$$
\Delta R_{i+1}=\frac{\partial R_{i+1}}{\partial L_{i}} \Delta L_{i}+\frac{\partial R_{i+1}}{\partial L_{i+1}} \Delta L_{i+1}+\frac{\partial R_{i+1}}{\partial R_{i}} \Delta R_{i}
$$

That is

$$
\Delta R_{i+1}=\frac{1}{2} \sqrt{\frac{f_{i+1}}{f_{i}}} \sqrt{\frac{1}{L_{i} L_{i+1}} R_{i}} \Delta L_{i}-\frac{1}{2} \sqrt{\frac{f_{i+1}}{f_{i}}} \sqrt{\frac{L_{i}}{L_{i+1}}} R_{i} \Delta L_{i+1}+\sqrt{\frac{f_{i+1}}{f_{i}}} \sqrt{\frac{L_{i}}{L_{i+1}}} \Delta R_{i}
$$

Hence, the relative error can be formulated as

$$
\frac{\Delta R_{i+1}}{R_{i+1}}=\frac{1}{2}\left(\sqrt{\frac{1}{L_{i}^{2}}} \Delta L_{i}-\sqrt{\frac{1}{L_{i+1}^{2}}} \Delta L_{i+1}+2 \frac{\Delta R_{i}}{R_{i}}\right)
$$

In accordance with the specification requirement, the ranging precision expressed by relative error is no more than $5 \%$, that is, as long as the following equation

$$
\left|\frac{\Delta R_{i+1}}{R_{i+1}}\right| \leq \frac{1}{2}\left|\frac{\Delta L_{i}}{L_{i}}\right|+\frac{1}{2}\left|\frac{\Delta L_{i+1}}{L_{i+1}}\right|+\left|\frac{\Delta R_{i}}{R_{i}}\right| \leq 5 \%
$$

is valid, specification requirement of relative location error can be achieved.

\section{SIMULATION EXPERIMENT ANALYSIS}

Suppose computer simulation is carried out to the location algorithm in existence of noise. Assume that the 
initial distance, initial velocity and sampling frequency are $R_{0}=8007.6 \mathrm{~m}, v_{0}=300 \mathrm{~m} / \mathrm{s}$ and $T=4 \mathrm{~Hz}$, respectively.

The relative measurement error of image size along the moving direction of the target is approximately $5 \%$ of pixel. The measurement error of angle is $\pm 1 \mathrm{mrad}$.

\section{A. Simulation experiment to a uniformly accelerated targe}

Suppose that the mobile target moves at an acceleration of $10 \mathrm{~m} / \mathrm{s}^{2}$ in a straight line. Fig. 3 shows the result of simulation experiment of mono-station passive location of a uniformly accelerated target in existence of noise. Fig. 3

$(3 a),(3 b),(3 c)$ are three dimensional motion model, motion speed, distance, the error between calculated distance and theoretical distance, respectively.

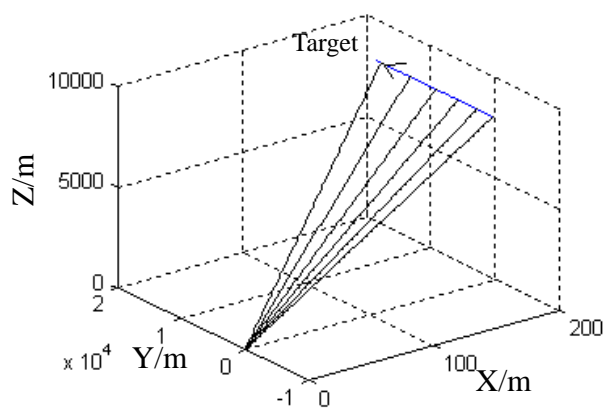

(3a) 3D motion model of a mobile target

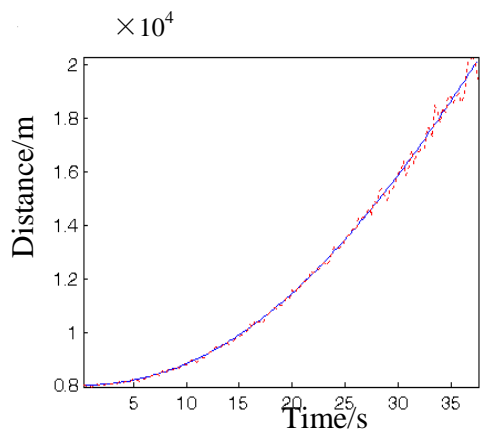

(3b) The distance of mobile target

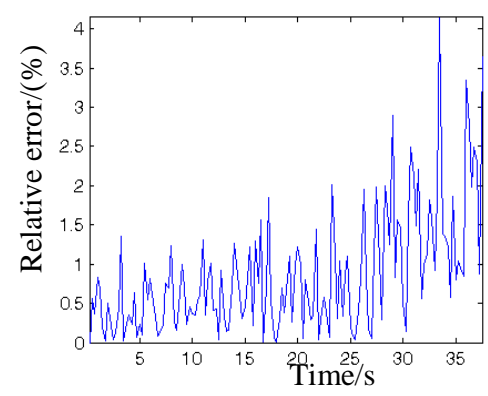

(3c) The rela. error of the distance

Fig. 3 Simulation results of a uniformly accelerated mobile target in existence of noise

\section{B. Simulation experiment of a target with varying acceleration}

Suppose that the mobile target moves at an acceleration of $a=[20 \sin (100 \pi t)+10] \mathrm{m} / \mathrm{s}^{2}$ in a straight line. Fig. 4 shows the results of simulation experiment of mono-station passive location of the target with changing acceleration in existence of noise. Fig. $4(4 a),(4 b)$,

$(4 c),(4 d)$ are three dimensional motion model, motion speed, distance, the error between calculated distance and theoretical distance, respectively, on this occasion.

Based on the simulation experiment above, we can see that the mono-station passive location algorithm to mobile targets due to stationary platform in this paper in existence of noise can be effectively applied to the location for both uniformly accelerated mobile target and that with varying acceleration. The relative ranging error is less than $5 \%$ of the slope distance from target to platform under the condition that the error of the target's parameter of image and angle is in permission.

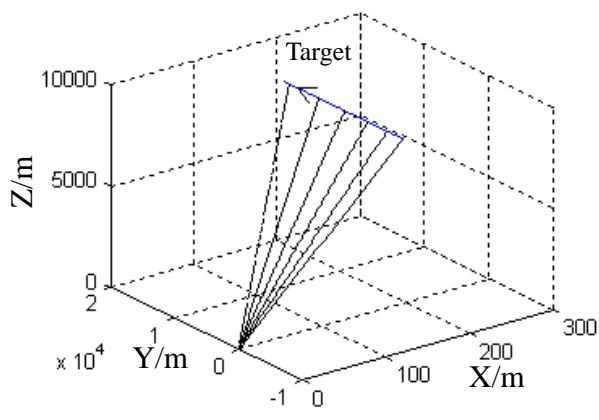

(4a) 3D motion model of a mobile target

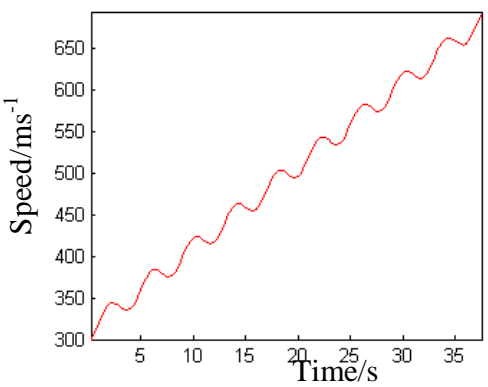

(4b) The speed of a mobile target 


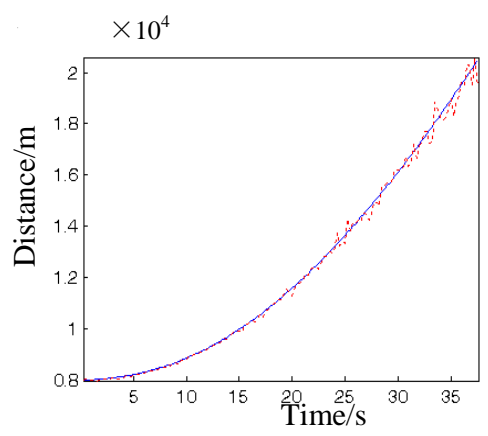

(4c) The distance of a mobile target

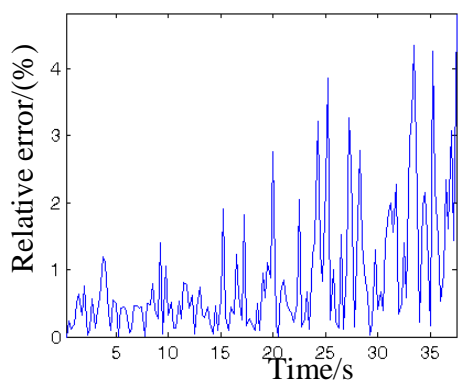

(4d) The relative error of the distance

Fig. 4 Simulation results of a mobile target with varying acceleration in existence of noise

\section{CONCLUSIONS}

Mathematical analysis and simulation experiments indicate that the mono-station passive location algorithm to mobile targets due to stationary platform established in this paper objectively reflects the actual situations of the measuring platform and the mobile target. The actual moving state and situation are objectively described in the algorithm since we take account of the imaging information along the moving direction and the information of the variation of the angle of course of the target. By doing so, the algorithm can overcome the passive effects of the pseudo-gesture variation of the mobile target on location precision. The mono-station passive location algorithm to the mobile target due to stationary platform in this paper can be effectively applied to the location for both uniformly accelerated mobile target and that with varying acceleration. The relative ranging error is less than $5 \%$ of the slope distance from the target to the platform, which completely satisfies the specification requirement of mono-station infrared passive location.

\section{ACKNOWLEDGMENT}

This work is supported by the National Natural Science Foundation of China (Grant No. 61072034), the Natural Science Foundation of China (Grant No. 61265006 ) and the Natural Science Foundation of China (Grant No. 51302318 )

\section{REFERENCES}

[1] J. P. Reilly, L. T. Youkins, R. J. Taylor, "Infrared passive ranging using sea background for accurate sensor registration," Proceedings of SPIE. 2469. pp. 319-329. 1995.

[2] J. P. Reilly, T. Kelein, H. Ilver, "Design and demonstration of an infrared passive ranging," John Hopkings APL Technical Digest. 20(2). pp. 1854-1859. 1999.

[3] Yin Shimin, Fu Xiaoning, Liu Shangqian, "The research for infrared mono-station passive location on stationary platform," Acta Photonica Sinica, 33(2), pp. 237-239. 2004.

[4] Cheng Bingwang., "Study on the new techniques of locating for multiple mobile targets on the basis of mono-station passive infrared method," Xidian University, Xi'an: Jan. 1998.

[5] Xiao Yang, "Passive locating techniques on the Basis of monostation passive infrared method," Xidian University, Xi'an, Jan. 2001.

[6] B. R. Xie, "The research on airborne infrared passive positioning method. Infrared Technology, ” 23(5), pp. 1-3, 2001.

[7] Z. T. Qian, "Passive ranging method for IRST systems," Journal of Infrared and Millimeter Waves. 20(4), pp. 311-314. 2004. 